\title{
MONITORING AND EVALUATING IMPLEMENTED HIS
}

by Kathleen A. McCormick, Ph.D.

National Institutes of Health, National Institute on Aging

Gerontology Research Center, Baltimore, MD 21224

\section{Abstract}

As early as 1973, Richard Nolan described a concept of stages in monitoring computer applications. By 1979 he refined his stages because of the experiences many companies in business had with computer proliferation. The stages that Nolan described may be applied to Hospital Information system dynamics. He described a time when an institution may undergo a transition from computer management to data resource management. It is a stage in the process of computer applications that evaluates the proposed change, considers the users new sophistication with the system, and alerts administrative personnel about the impact of proposed changes. This paper presents an organizational concept for monitoring 20 elements when considering HIS changes so that unilateral changes that are uncoordinated can be avoided. When a hospital reaches a stage where changes in the system, both negative and positive can be monitored and evaluated, the organization is entering a stage of maturity.

When Richard Nolan described a "stage hypothesis" in 1973 for managing the computer resource, he proposed that controlling the computer as a resource over time does change in character ${ }^{1}$.

From over a decade of experience with computers in business settings, he described a stage pattern leading to computer management principles. Stage 1 was Initiation which is brought about by the acquisition of the computer into the organization. During this time the organization and administrator recognize that the computer is going to force organizational change. Stage II was Contagion. This stage is characterized by management encouraging users to develop content for the computer. once a few office rumors indicate that the computer is capable of time-saving feats, the requests for computer usage become saturated. Without a carefully designed implementation plan, run away costs and content begin to accrue during this stage. Stage III was Control, a stage which was entered due to the crisis of contagion. At this time a centralized computer facility may be formed, expenditures are analyzed, inefficiencies in computer applications are described, and a special steering committee is formed to redesign the implementation plan. The result is a high level of competition for relatively scarce computer resources including personnel, technical support, money, and hardware. Stage IV was an Integration Stage. The most dominant characteristic of this stage is the rethinking about the input into the computer. The computer's processing capabilities, and the desired output of the computer. The computer is linked to organizational mission, goals and objectives while staying within a budget for the first time within stage IV.

By 1979 Richard Nolan analyzed the formative stages of computers in the business world again and expanded his stages to $\mathrm{six}^{2}$. The four stages continued to be valid, but experience unveiled a larger concept. Stage I remained a stage of Initiation, Stage II that of contagion and stage III Control. During these stages the management skills are needed to manage the computer as a new resource in the organization. Stage IV was still defined as Integration which brought about a change in management to management of the data resources. In addition, this shift in emphasis on management brought about restructuring in the organization, and two new stages that had previously been obscured by development and implementation trends became apparent. Stage $\mathrm{V}$ was defined as Data Administration which was characterized by the controls that management could expect from the computer output. For example, reports representing multiple layers of the organization could be generated hourly, daily, weekly, monthly, quarterly or annually. Doing business with other companies became feasible through the micro-computers. Companies could buy inventory from industry, witharaw money from banks, or schedule airline tickets using the companies computer. The last stage, Stage VI that Nolan identified was Maturity, a stage which was reached when the applications for the computer are complete and its structure "mirrors" the organization and the information flows in the company.

\section{Applications to Hospital Information systems}

In their formative years, hospital information system managers and hospital administrators can perhaps identify with the Stage Hypothesis that Nolan described for business and industry. A few hospitals that have had over a decade of experience with computers may also recognize that no hospital information system remains adequate forever. Even with the most elegant PERT plan for planning, implementing, designing and evaluating the system that you invested in, some hospitals have reached a stage of Maturity.

It is known from the literature that 
extensive computer applications have been described in hospitals ${ }^{3}$. It is also known that once developed the content in computer matrices may be extensive, overlapping, and not used efficiently ${ }^{4}$. similarly, users attitudes and level of sophistication change. In order then to manage the data resource efficiently, criterion need to be monitored to determine content that should be deleted, added, changed, or evaluated in a formal study. This becomes a continuous process involving evaluation and management experts to evaluate desirous changes in the hospital information system.

A flow-chart model of ongoing system evaluation was described by Schmitz in his book on HIS in $1979^{5}$. This model described the numerous issues that may contribute to weighing decisions about changes in the computer. Basically, in this model the re-evaluation may be due to: 1) changes in the legal environment, 2) changes in the consumer evnironment, 3) changes in the regulatory environment, and 4) changes in the internal organization. The purpose of the evaluation, he describes as facilitating decisions to: modify the software, modify the hardware, retrain the users, or scrap the system.

\section{Twenty Elements for Monitoring HIS}

From this author's experience, there appears to be twenty elements for monitoring and evaluating change for a more functional Hospital Information system (HIS). These elements could be review criterion used by the information systems department, nurse administrators, and hospital administrators following a formal request for a change in the system. These elements do not justify the lack of systematically evaluating and monitoring problems with the system, increased user sophistication, and recognition of expanded system capabilities in response to changeing hospital requirements and advances in computer technology. These elements do facilitate monitoring and evaluating the complex issues that arise in managing hospital information systems which produce data resources. A description of 20 elements follows :

1. The System Request Form is Complete. Each hospital should develop a form that may be used to define a proposed change in the system or identify a problem with the system. This proposal format is in addition to a log-book that contains information on down-time, technical problems with keyboards and printers, and ongoing costs. The form should contain appropriate questions to generate the information that is needed as follows.

2. The Type of Change Should be Identified. It does not matter how well planned the computer system was in the design and implementation phases, changes may be required in the software and hardware. These are serious and costly problems that involve technical support and professional input. An example might be the "flagging" of all error entries into the system rather than "flagging" only medication errors while allowing text-editing capabilities for other type-in errors.
A change in users attitudes about the computer can result in requests for flexibility in the content area, a new category, the ability to print additional information, and the deletion of content not used.

A change in user sophistication can result in requests to change the input format or the printed output reports. Changes due to user sophistication might include a change in the screen sequencing, the layout of screens graphically, or the introduction of more pertinent information on screens.

3. The Purpose of the Change is Identified. When a formal proposal is received to make a change in a computer system the author of the proposal must be able to clearly state the purpose of the change. It is then evident to the reviewers whether the applicant intends a positive or negative outcome from the proposed change. By stating the purpose clearly, it will be evident whether the change reflects new developments that have occurred in the hospital organization or the technology industry. The appropriateness of the change, its impact on quality and quantity, and its costs related to benefits are all weighted in the purpose.

4. The Change Meets Legal Documentation Requirements. Because the computer must be flexible to accomodate changes, consideration of the potential changes resultant from legal sources must be made when purchasing an information system. For example, if new nurse practice acts in the state require that registered nurses write a "progress note" every time a physician sees a patient, this legal documentation requirement must be added to the computer. Similarly, if malpractice insurance coverage was dependent upon physicians justifying diagnostic or therapeutic orders, content within the computer would have to be changed.

5. The Change is Consistent with Professional/Institutional standards. The ability of the computer to input, process, and output records which document communication within a hospital, patient care given, and summarize procedural results make it important that the format can adapt to changes in standards. Professional standards for nursing which were published by the American Nurses' Association could be within the computer content, however, if specialty organizations develop specific standards for patients with specific diseases, then the institution must determine which standards will be adhered to by the computer-assisted-documentation.

6. The Change Results from Changes in the Consumer/Patient Environment. Increased public attention to malpractice, the efficacy of medical devices, self-care, consumer participation in health care, and patient's rights make it important to plan specific content within the computer for the patient. Informed consent documentation may have been neglected in the original design and implementation of the computer, but with more user sophistication the requests for 
incluuing documentation of signed informed consents may reach the information system office. As more nursing standards include justification of the patient's involvement in care planning, patient screens may be needed in the menu selection.

7. The Proposed Change is Consistent with program Goals. When planning, designing and implementing a computer system, objectives are usually written consistent with progran goals. However, any dynamic organization with flexible retention of leadership, experiences program goal changes regularly. Therefore, in considering changes in the computer content or system it is important that the review process establishes a management feedback and balance between developments and program goals. If management does not establish a balance between program goals and computer capabilities, budgeted resources can be allocated for competing concepts. For example, a major inservice education program can be allocated for teaching nursing personnel primary nursing process and documentation, however, a major computer program can be allocated to establish nursing documentation of functional tasks only.

8. The Proposed Change fits a Documented Theoretical Format. The important function of monitoring this aspect becomes evident when the output of information in Hospital Information Systems may be used for research data. For example, if a documentation capability of the computer is to describe patient's pain perceptions, there are available theoretical frameworks for assessing pain as a quality which has quantity, location, and duration.

9. The Proposed Change improves Quality Control/Validity/Reliability/Risk Management. Hospital organizations are made up of interrelated departments with interrelated monitoring controls. Changes in one part of a HIS for research retrieval is going to impact on another part of the system for quality control and/or risk management. Unless a proposed change includes content that is valid and produces reliable information, the computer risks storing "data cemeteries". It is therefore desirable to consider changes in a computer in groups or batches to avoid duplication in cost, in time, and in effort. The greatest savings in the management of HIS changes occurs when the changes are grouped logically. This grouping allows a priority rank to be assigned to certain change requests, giving priority to changes that impact upon quality Control, Research Retrieval, and Risk Management. Scheduled changes in a computer system should be more cost effective to a hospital than responding to every request that users make.

10. The Proposed Change Avoids Duplicating Existing Information. When the initiated computer system has progressed through the contagion stage, the likelihood of duplication is great. Specialty groups begin to increase specialty software for their particular needs. Without some form of centralized monitoring of the content being developed for the HIS, duplication of content, costs, time and effort results. This area again highlights the need for management of the data resources. If the hospital management abdicates control of the computer content to the technical personnel, key problems like duplicating content cannot be avoided. If, however, management personnel manage the data resources, some problems can be avoided.

11. The Proposed Change Incorporates Advances in Information System Technology. Computer equipment is outdated so quickly that new technology is perhaps available before a hospital has fully implemented their system. While it is never recommended that implementation be interrupted once it has begun, there are potential technological advances that are occurring that implementors must not lose sight of. This concept makes the purchase of a system so important, since a system must be flexible enough to include compatibility with new hardware, new. software, and new uses of data resources. Vigilant monitoring of new technological developments is needed by persons monitoring changes, managing hospitals, and implementing systems.

12. The Proposed Change Meets Federal and State Regulatory Requirements. In the area of third-party reimbursement, certain documentation requirements exist. However, regulatory agencies frequently change documentation requirements. If the process has been computerized, a flexible system can incorporate these changes. If a change in State Regulatory Requirements is the only justification for a proposed change in a computer system, it will undoubtedly receive a high priority ranking. Flexible computer systems are responding to "DRG" requirements.

13. An Evaluation Mechanism is Identified with the Proposal. Unless a proposed change includes a feedback mechanism to the review group, who would ever know if the proposed change met its desired impact? For example, changes can occur in a system unnoticed, not used, never improve efficiency, and essentially waste money, time and effort. Evaluation mechanisms may include a pilot study, quarterly or annual reports, or the recommendation of a major evaluation study. If the purpose of a proposed change was to provide user accessibility to content for better documentation, a realistic evaluation monitor would be a before and after report on the usage of the content changed.

14. The Proposed Change Impacts on the User. User impact may be the most significant justification for a change in a system. If a proposed change can document decreased user work in entering data, retrieving data, finding data, and/or accessing data, users usually become more satisfied with the new technology. As high technology influences our organizations more, allowing flexible changes in the computer to pacify the user adds the high touch or humanism to the balance. It should be pointed out that users may unilaterally solicit changes that are in 
opposition to each other, are negative to the system goals, and are generally not positive additions to the system. Often the data resource managers are faced with challenging user attitudes with program goals.

15. The Proposed Change Impacts on the Output Capability and Retrieval Capability without Interfering with Security. Unplanned and uncoordinated content may be added to a system which cannot be retrieved within an hour, a day, a week, or even a month. Data content changes often necessitate coding format changes. Data content changes also pose a threat of interfering with the security of the data. The proposed change should not be given a priority if the output data would be erroneous or missing. The proposed change must also be considered in light of access, that is, whether access in on-line, off-line, for specific persons only, and if data needs updating.

16. Costs are Identified. Changes in a system during or after implementation costs resources: technical, professional and equipment; which costs money. Few HIS changes merit diverting monetary resources from program budgets. Many proposed changes are not essential to the operation of the organization. Any hospital with a HIS needs a budget for maintenance costs which should include change costs. Changes often require resources that differ from implementation resources. For example, in implementation phases the initiators are usually computer systems analysts and programmers. In the change phase, the resources may be consultants, hardware, software, and researchers.

\section{Dissemination Requirements are} Identified. If a change is proposed that will impact upon the entire nursing staff, how does the applicant plan on disseminating the information about the change? Are new policies required? will memorandum be used? will flashing screens be necessary? Will an individualized computer telegram be sufficient?

18. Training Program Requirements are Identified. Some changes in hospital information systems will be congruent with other program goals. However, special training needs may exist due to a proposed change. The cost, time, and trouble in implementing a proposed change that requires training doubles, since inservice education programs involve additional personnel and time.

19. The Flexibility of the Proposed Change is Identified without Sacrificing Security. Just as flexibility is important in the purchase of a computer, so too is the flexibility of the change important. The introduction of a change should be approached with the utmost assurance of the compatibility of the change with the old system. The compatibility of a change with current operations is often the controlling factor in the decision as to whether the change can be made.

20. The Availability of Resources to Accomplish the Change Are Identified. Resources include personnel, equipment, software, travel and other budgets. Personnel may be technical, professional or consultant staff. It would be fruitless to recommend a proposed change that the current hardware cannot accommodate. New computer software may be available to implement the proposed change, but may be incompatible with the system in use at the hospital.

\section{Summary}

When a hospital decides to purchase a computer, it has made a commitment to change. When a hospital reaches a stage where changes in the system, both negative and positive can be monitored and evaluated, the organizational process is entering a stage of maturity. The priorities of managing the computer must shift to managing the data resources. A system of feedback with subsequent impact on the entire hospital organization must be established.

\section{References}

1

Nolan, Richard. "Managing the Computer Resource: A Stage Hypothesis," Communications of the ACM, 16:399-405, 1973.

Nolan, Richard. "Managing the Crises in Data Processing," Harvard Business Review, 115126, March-April, 1979.

3

Bronzino, Joseph. Computer Applications for Patient Care. Reading, Massachusetts: Addison-Wesley Publishing Co., 1982.

4 Romano, C., McCormick, K., and McNeely, I. "Nursing Documentation: A Model for a Computerized Data Base," Advances in Nursing Science, 4:43-56, January, 1982.

5 Schmitz, Homer. Hospital Information systems. Aspen System Corp., 1979. 